\title{
Titolo esteso:
}

Tactical Emergency Casualty Care . Medicina tattica o nuovo approccio al soccorso sanitario ordinario?

\section{Autori:}

Dott. Nicola Bortoli; Medico Anestesista Rianimatore - Dirigente Medico U.O.C. Centrale Operativa 118 SUEM di Venezia

Dott. Luca Panizza; Infermiere presso U.O.C. Centrale Operativa 118 SUEM di Venezia U.O.S. SUEM Venezia

\section{Abstract}

Dall'11 settembre 2001 al 31 dicembre 2017, nel mondo, sono stati documentati 108.374 attacchi terroristici. La maggior parte delle lesioni riscontrate sui soggetti coinvolti sono assimilabili a quelle rinvenute nei teatri bellici. Il 25\% delle vittime possono essere salvate con misure salvavita appropriate se attuate immediatamente già sul sito dell'attentato adottando procedure di intervento come quelle proposte dal Tactical Emergency Casualty Care. E' stato condotto uno studio osservazionale trasversale che mettesse a confronto le competenze acquisite da professionisti che hanno partecipato ad un corso di medicina tattica, secondo le linee guida TCCC/TECC, e professionisti che hanno frequentato un corso di gestione del trauma secondo linee guida ATLS/PHTC. Obiettivi secondari dello studio miravano a verificare se queste competenze fossero state utilizzate, dagli stessi professionisti, nelle attività di soccorso al paziente traumatizzato in un contesto ordinario.

Lo studio è stato realizzato mediante somministrazione di un questionario ad un campione di 154 professionisti (medici, infermieri ed autisti soccorritori) del servizio di emergenzaurgenza 118 italiano.

I partecipanti allo studio che hanno preso parte ad un corso di medicina tattica ammontavano al 30\% del campione. Lo studio ha evidenziato che il $60 \%$ dei professionisti appartenenti al gruppo che ha partecipato a corsi TCCC o TECC poneva maggior attenzione al controllo delle emorragie e ad una precoce stabilizzazione delle vie aeree rispetto al restante campione di studio.

KEYWORDS: TECC, Tactical medicine, Prehospital trauma, terrorist attack, TCCC 


\section{INTRODUZIONE}

Gli anni 2000 hanno registrato un incremento degli eventi emergenziali riconducibili ad atti di matrice terroristica. Tra gli eventi più emblematici si annoverano: la bomba al Murrah Building nel 1993 (Oklahoma City), l'attentato al Pentagono del 2001 (Washington) e al World Trade Center del 1993 e soprattutto dell'11 settembre 2001 (New York City) che segnarono un profondo cambiamento degli equilibri geopolitici mondiali. In Europa, sono seguiti ulteriori attentati tra cui si ricordano: Madrid dell'11 marzo 2004 (attacchi al sistema ferroviario; 191 morti e 2057 feriti); Londra del 7 luglio 2005 (esplosioni contro il sistema di trasporti pubblici durante l'ora di punta; 52 morti e circa 700 feriti); Parigi nel 2015 (serie di attentati con l'uso di armi da fuoco; 130 morti e più di 350 feriti); Nizza nel 2016 (singolo attentatore alla guida di un autocarro lungo la Promenade des Anglais (86 deceduti e 302 feriti). ${ }^{1-4}$

Dall'11 settembre 2001 al 31 dicembre 2017, nel mondo, sono stati registrati 108.374 attacchi terroristici, dei quali 575 nel Nord America, 2726 in Europa Occidentale, di cui 106 in Italia. Il 59\% degli attacchi ha visto l'utilizzo di ordigni esplosivi mentre un 33\% armi da fuoco. ${ }^{2}$ Dalla revisione di questi eventi è emerso che la maggior parte delle lesioni riscontrate sulle vittime erano assimilabili a quelle riscontrate in teatro bellico e rappresentate prevalentemente da traumi penetranti al torace, all'addome o traumi da esplosione (blast injuries). ${ }^{3-5}$

L'unico aspetto significativamente differente tra i traumi appena descritti in contesti civili ed i paritetici riscontrabili nei teatri di operazione militari è rappresentato dalla diversa tipologia di vittime coinvolte. Il personale militare, per sua natura, è caratterizzato da soggetti tendenzialmente giovani, in buona salute e che, al momento del trauma, indossa specifici dispositivi di difesa individuale (elmetto, giubbotto antiproiettile, etc.) e protetto ulteriormente da sistemi di difesa collettiva (ad esempio, veicoli blindati/corazzati). Le vittime di attentati terroristici in scenari urbani, invece sono rappresentate da civili con età estremamente variabile (da infanti ad anziani), spesso portatori di patologie croniche e in assenza di dispositivi di protezione al momento del trauma. ${ }^{6}$

Il Tactical Combat Casualty Care (TCCC) consiste in un approccio alle vittime sul campo di battaglia che prende vita nella seconda metà degli anni 90 dal PHTLS (PreHospital Trauma Life Support) della National Association of Emergency Medical Technicians (NAEMT). Il TCCC rappresenta il protocollo ufficiale di gestione dei traumi da parte dell'esercito statunitense, del Comando unificato delle forze armate americane (USSOCOM) e dagli eserciti della coalizione NATO. ${ }^{7,8}$ 
Secondo il TCCC l'approccio valutativo al ferito viene effettuato secondo l'algoritmo MARCH-PAWS: (M) gestione delle emorragie massive tramite l'utilizzo di tourniquet, garze emostatiche e presidi giunzionali; (A) gestione delle vie aeree; (R) gestione della respirazione; (C) gestione dello shock; $(\mathrm{H})$ gestione dell'ipotermia e dei traumatismi del capo; (P) gestione del dolore con l'utilizzo di FANS, Fentanyl OTFC o Fentanyl/Ketamina IV o IM; (A) antibioticoterapia precoce per OS, IV/IO/IM in tutte le condizioni con ferite aperte; (W) pulizia e medicazione delle ferite; (S) immobilizzazione mediante utilizzo di stecche 0 barelle. ${ }^{9,10}$

Il Tactical Emergency Casualty Care (TECC), invece, nasce nel 2011 come protocollo redatto per i professionisti del Soccorso e della Sicurezza addestrati a soccorrere vittime di trauma violento, in ambiente tattico civile o socialmente pericoloso, al fine di garantire risposte operative efficaci in occasione di eventi come sparatorie, attentati o attacchi terroristici. Sviluppato anch'esso dal Comitato del PHTLS della NAEMT, si basa sulle Linee Guida del CoTCCC (Tactical Combat Casualty Care Committee), adattando gli insegnamenti appresi in ambito militare, relativi al trattamento dei feriti in combattimento, e applicandoli alla medicina tattica nel contesto civile.

Pariteticamente al TCCC, anche il TECC riconosce tre fasi ben distinte di intervento:

1. Direct Threat Care (Soccorso durante Minaccia Diretto): insieme di manovre di soccorso erogate mentre si è sotto attacco o in condizioni non permissive;

2. Indirect Threat Care (Soccorso durante Minaccia Indiretto): il soccorso fornito mentre il pericolo è stato neutralizzato in maniera definitiva o temporanea, con la possibilità che si ripresenti;

3. Evacuation (Soccorso durante Evacuazione): il soccorso fornito mentre il ferito viene allontanato dal sito dell'incidente e diretto verso in luogo di cure di livello superiore.

Il TECC approccia al ferito con lo scopo di ridurre la mortalità da lesioni tipiche di contesti tattici attraverso strategie miranti: al controllo delle emorragie, al controllo chirurgico delle vie aeree, alla decompressione dello pneumotorace iperteso, al posizionamento di accessi venosi ed intraossei, ad adeguate strategie per il trattamento di soccorritori feriti in ambienti pericolosi e di pazienti pediatrici, nonchè al mettere in pratica tecniche per il trasporto e la movimentazione dei feriti. ${ }^{11-13}$ 


\section{Materiali e metodi}

Dalla letteratura emerge che le vittime di sparatorie, attentati terroristici, traumi derivanti da incidenti automobilistici e da violenza criminale possono essere salvate con l'uso di concetti TCCC adattati al contesto civile. ${ }^{14,15}$ Gli attuali protocolli civili enfatizzano l'importanza della sicurezza personale dell'operatore, ma non sono in grado di fornire un'analisi basata sull'evidenza dei pericoli sulla scena relativi agli incidenti terroristici. Vi è la necessità di affinare le indicazioni di sicurezza per i soccorritori sanitari alla luce dell'esperienza legata alle migliaia di incidenti che si verificano ogni anno a livello globale. ${ }^{16}$ L'Hartford Consensus ed il CoTECC (Committee for Tactical Emergency Casualty Care), nelle loro raccomandazioni, sottolineano l'importanza della formazione estesa alla popolazione generale per migliorare la risposta ad eventi critici. Tenendo conto dell'esperienza del bombardamento nella maratona di Boston, dove l'applicazione di tourniquet hanno salvato numerose vittime, si suggerisce la costituzione di squadre integrate (forze di polizia, vigili del fuoco, personale sanitario); viceversa, non ci sono evidenze che sconsiglino l'integrazione di un'attenzione tattica all'emergenza civile. ${ }^{17-20}$

L'esperienza acquisita nel corso dell'ultimo decennio ha dimostrato che, per avere delle significative riduzioni della mortalità, è necessario un trattamento precoce sulla scena del ferimento. Gli operatori, a tutti i livelli, dovrebbero essere dotati di tourniquet ed essere addestrati alla gestione dei traumi penetranti da armi da fuoco. ${ }^{8,19}$

Il tourniquet è uno strumento salva-vita facilmente impiegabile che può essere utilizzato per controllare l'emorragia degli arti. Molte informazioni fuorvianti nel corso degli anni hanno portato ad una progressiva sfiducia nei suoi confronti. Tuttavia, queste informazioni sono state tramandate da un'epoca in cui le cure definitive erano molto ritardate o subottimali. Studi recenti ed il suo impiego in setting militari dimostrano elevati livelli di sicurezza con una chiara raccomandazione all'ampliamento del suo impiego anche all'ambito civile. ${ }^{21}$ L'utilizzo del Combat Application Tourniquet (CAT) ha dimostrato un'efficacia che va dal $78 \%$ al $100 \%$ e un'incidenza di complicanze ad esso associate $<2 \%$. L'utilizzo del presidio risulta facile e rapido. Il posizionamento in sede per 2 ore consecutive non risulta associato a danni permanenti degli arti. È necessario porre attenzione al suo utilizzo su pazienti anziani, pediatrici o con comorbidità. Le situazioni in cui un CAT potrebbe essere applicato per arrestare l'emorragia dell'estremità sono numerose nel setting civile comprese le minacce crescenti legate agli attacchi terroristici o nelle condizioni di maxi-emergenza. ${ }^{22-24}$

Il CoTCCC, inoltre, raccomanda l'impiego di presidi ad azione emostatica per il controllo delle emorragie in sedi anatomiche nelle quali non è possibile posizionare un tourniquet 
(collo, ascella, inguine). Studi riguardanti l'uso degli agenti emostatici di prima generazione come l'HemCon hanno ottenuto un tasso di successo che va dal $74 \%$ al $97 \%$ nell'arrestare l'emorragia. Studi condotti sull'utilizzo del QuikClot di prima generazione hanno segnalato una efficacia del $92 \%$, riscontrando buona efficacia anche in setting civili come dimostrato negli Stati Uniti e nel Regno Unito. ${ }^{25-29}$

La gestione delle vie aeree nel TCCC prevede sia l'impiego di tecniche tradizionali di base che avanzate, come la cricotiroidotomia. I tassi di incidenza di cricotiroidotomia in ambito militare sono il doppio rispetto all'ambito civile. Ciò è dovuto all'elevata incidenza di traumi penetranti (frammenti) prodotti da ordigni esplosivi improvvisati, ferite da arma da fuoco, nonché ustioni delle alte vie aeree. ${ }^{30,31}$ I traumi toracici rappresentano il $25 \%$ delle cause di decesso a seguito di lesioni aperte o chiuse. Le lesioni aperte solitamente sono provocate da punta, punta taglio, come armi bianche o altri oggetti o da armi da fuoco o schegge. Le lesioni chiuse solitamente sono prodotte da impatti del corpo contro ostacoli fissi o inferte da oggetti contundenti o come conseguenza dell'onda d'urto prodotte da un'esplosione. Circa il 30\% dei traumi toracici aperti ed il 10\% di quelli chiusi richiedono il trattamento chirurgico d'urgenza. La condizione di pneumotorace iperteso (lesioni chiuse) costituisce una condizione che può portare a decesso se non riconosciuto e trattato precocemente già sul territorio mediante puntura decompressiva con ago o, ancor meglio, con il posizionamento di un drenaggio toracico ${ }^{9}$.

L'accesso venoso rapido è un presupposto fondamentale per la gestione del paziente traumatico ed in peri-arresto. La difficoltà nel reperimento di un accesso venoso periferico nel paziente in shock ipovolemico risulta proporzionale alla gravità della situazione. L'accesso intraosseo ha dimostrato di essere efficace quanto altri accessi con la possibilità di infondere grandi volumi di liquidi con effetti paragonabili all'accesso venoso periferico, sia in termini di farmacodinamica che di farmacocinetica. ${ }^{22,32,33}$ Le conoscenze riguardanti il riempimento volemico pre-ospedaliero delle vittime emorragiche nel 1992 indicavano di somministrare 2 litri di soluzione salina il più rapidamente possibile. Una revisione della letteratura ha indicato che questa raccomandazione non era stata sostenuta da prove di efficacia. Da allora il CoTCCC ha introdotto il concetto di ipotensione permissiva. L'ipotensione permissiva è un concetto emergente nella cura del trauma, dove si infondono liquidi per raggiungere dei target pressori inferiori ai valori normali per limitare il sanguinamento, escludendo i pazienti con una lesione celebrale traumatica, al fine di non creare un danno secondario dovuto all'ipoperfusione. ${ }^{34-37}$

Durante i conflitti in Iraq e Afganistan il 14\% dei feriti ricoverati presso i ROLE 3 hanno ricevuto almeno una trasfusione di emocomponenti. Di questi il $35 \%$ ha ricevuto trasfusioni 
massive, intese come $>10$ unità di emazie concentrate o sangue intero in 24 ore. La Damage Control Resuscitation (DCR) rappresenta una strategia complementare alla Damage Control Surgery (DCS) che consiste nel trattare esclusivamente le lesioni chirurgiche potenzialmente letali posticipando il trattamento definitivo al trattamento delle alterazioni metaboliche e fisiologiche in grado di sviluppare complicanze post chirurgiche gravi. La DCR consente di dare priorità agli interventi non chirurgici in grado di ridurre morbilità e mortalità mediante il ripristino dell'omeostasi, la prevenzione o la mitigazione dello sviluppo di ipossia tissutale, debito d'ossigeno, ipoperfusione e coagulopatia. L'obiettivo viene ottenuto al meglio mediante un controllo aggressivo dell'emorragia e la rianimazione basata su emoderivati in grado di ripristinare l'ossigenazione dei tessuti, evitare l'emodiluizione di piastrine e dei fattori della coagulazione provvedendo alla sostituzione del potenziale emostatico perso con l'emorragia. La DCR risulta maggiormente efficace con l'utilizzo di sangue intero ${ }^{4142434445}$.

\section{METODO}

E' stato condotto uno studio osservazionale di tipo quantitativo, con disegno descrittivo a campionamento non probabilistico di convenienza.

Lo scopo primario dello studio è stato quello di mettere a confronto le competenze acquisite dai professionisti dei servizi di emergenza edurgenza extra-ospedaliera che hanno partecipato ad un corso di "medicina tattica", secondo le linee guida TCCC o TECC, con un gruppo di professionisti che non ha partecipato a tali corsi. Obiettivi secondari sono stati rappresentati (1) dalla verifica di eventuale impiego delle conoscenze acquisite durante un corso di medicina tattica in occasione di interventi ordinari in ambito civile e (2) la presenza di eventuali bisogni formativi in merito. Ė stata utilizzato un campionamento di convenienza contattando Medici, Infermieri, Autisti soccorritori che lavoravano nei servizi di emergenza extra-ospedalieri nel territorio italiano.

Il questionario utilizzato era uno strumento ex-novo. Era composto da 16 domande a risposta singola o multipla suddivise in:

- 5 quesiti sociodemografici;

- 6 di core competences legate alle linee guida TECC;

- 2 indaganti la realtà lavorativa;

- 2 relative ai fabbisogni formativi;

- 1 domanda di valutazione questionario riguardante 3 items.

I dati raccolti sono stati acquisiti ed elaborati mediante l'uso della piattaforma internet 
SURVIO ${ }^{38}{ }^{38}$, per l'ulteriore elaborazione dei dati è stato utilizzato il programma EXCEL ${ }^{\circledR}$ di Microsoft ${ }^{\circledR}$.

Il 63\% dei partecipanti ha impiegato un tempo tra i 2 e i 5 minuti per la compilazione del questionario mentre il $29.2 \%$ dai 5 ai 10 minuti. È stato garantito l'anonimato a tutti i partecipanti e l'adesione è stata volontaria.

\section{RISULTATI}

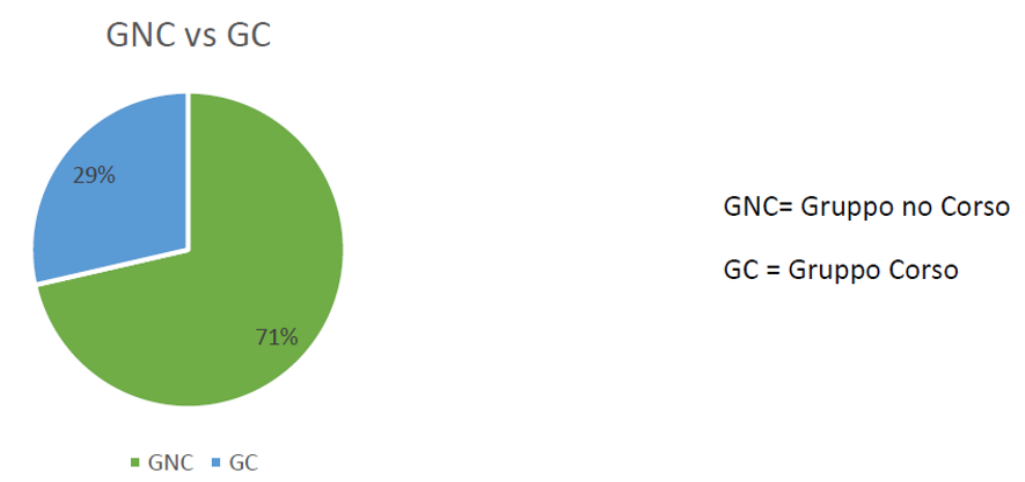

Il questionario è stato compilato da 154 professionisti così ripartiti: 28 medici, 82 infermieri, 44 autisti soccorritori. L'anzianità lavorativa va da 1 a 5 anni per 48 professionisti, da 6 a 10 anni per 33 professionisti e superiore a 10 anni per 73 partecipanti. Quindici intervistati hanno dichiarato di avere una specializzazione in Anestesia e Rianimazione o Medicina emergenza-urgenza, 28 un Master in Area Critica, 111 di essere solo in possesso delle certificazioni d'obbligo previste dalla propria regione di servizio. Degli intervistati 106 prestano servizio al Nord, 37 al Centro, 11 in Sud e Isole.

\section{Professione Numero compilazioni}

Medico 28

Infermiere $\quad 82$

Autista soccorritore 44

\section{Anzianità lavorativa Professionisti}

$\begin{array}{ll}1-5 \text { anni } & 48 \\ 6-10 \text { anni } & 33 \\ \text { > a 10 anni } & 73\end{array}$

Relativamente alla partecipazione a corsi di medicina tattica in 8 hanno indicato la 
partecipazione al corso TEMS (Tactical Emergency Medical Service), 12 al corso TCCC, 7 al corso TECC, 14 ad un corso di Medicina Tattica, 23 al corso Advanced Combat Life Support, 90 nessun corso. Trenta intervistati hanno dichiarato di aver frequentato altre tipologie di corso, tra i quali: i corsi di Bleeding Control e il Corso NISS gestito dal 118 di Vicenza.

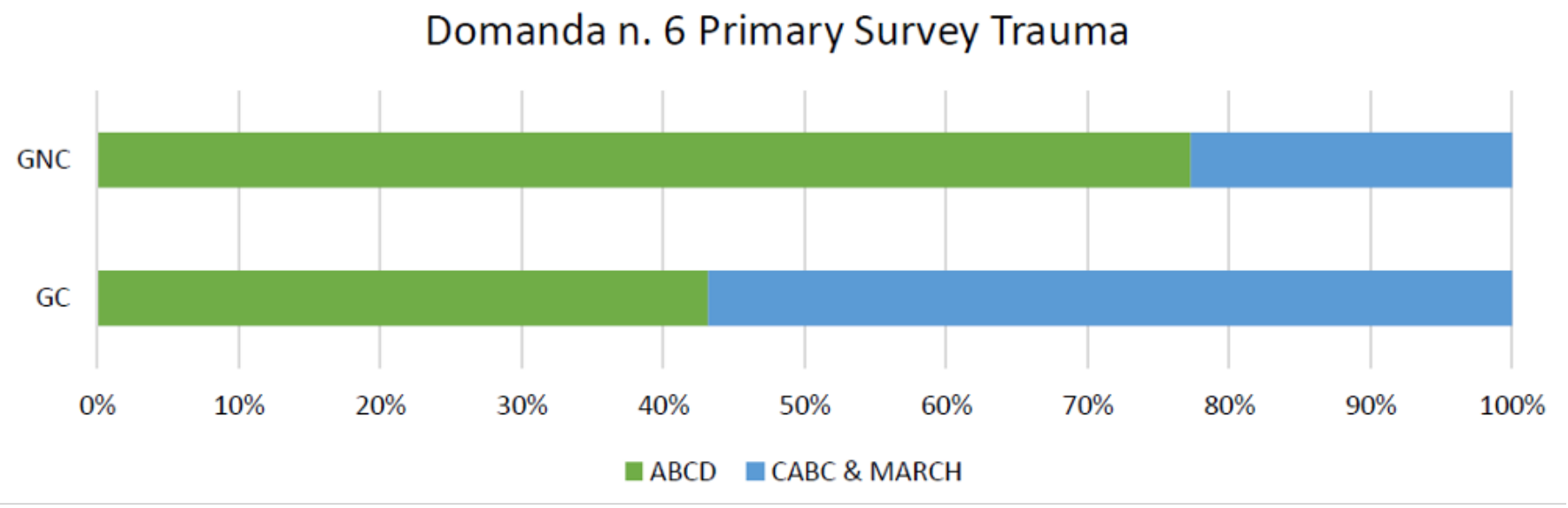

\section{Relativamente ai quesiti sulle core competences in ambito di medicina tattica, le risposte ottenute sono rappresentate nella Tabella:}

- nella primary survey del paziente traumatico quale dei seguenti acronimi ritiene più corretto? - l'acronimo ABCD viene considerato corretto da 104 persone, il CABC da 25, il MARCH da 25;

- in caso di emorragia massiva ad un arto cosa farebbe/posizionerebbe come prima azione? - il bendaggio compressivo "classico" viene scelto da 23 persone, 62 tamponamento diretto della ferita e 68 sceglie l'uso del tourniquet;

- in caso di trauma penetrante all'addome cosa farebbe/posizionerebbe come prima azione? - 31 persone scelgono il bendaggio compressivo, 79 il tamponamento diretto sulla ferita e 44 la benda istreliana;

- in causa di trauma penetrante al torace cosa farebbe/posizionerebbe come prima azione? - 88 applicherebbero una medicazione chiusa sui tre lati, 56 una medicazione con valvola e 10 una garza grassa;

- in presenza di pneumotorace chiuso cosa farebbe/posizionerebbe come prima azione? - 88 risposte sono state date a favore della decompressione con ago da 14g, 29 mediante l'uso di needle kit e 37 attenderebbero l'instabilità dei parametri vitali prima di intervenire;

- in caso di ostruzione delle vie aeree cosa farebbe/posizionerebbe come prima azione? 52 persone hanno risposto cannula orofaringea, 18 intubazione orotracheale, 52 Crico 
Kit, 32 cricotiroidotomia con ago.

Domanda n. 7 Gestione emorragia massiva arto

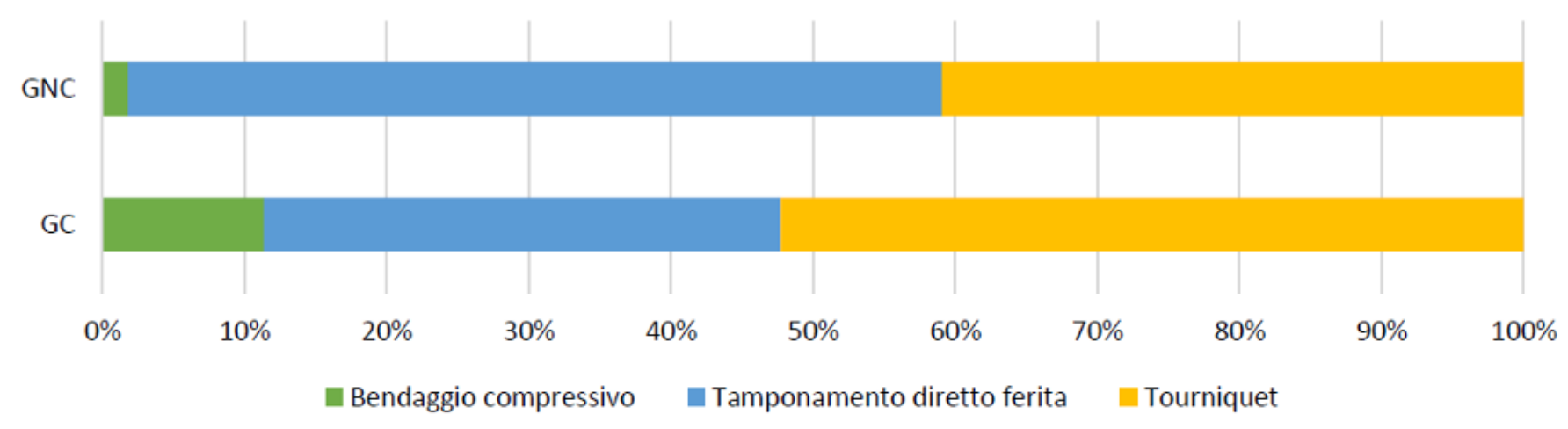

Indagando l'utilizzo dei presidi visionati nei corsi di medicina tattica 47 persone riferiscono di aver utilizzato il tourniquet nel loro lavoro ordinario, 19 l'emergency bendage, 20 le medicazioni emostatiche, 17 il Crico Kit, mentre 82 dichiarano di non aver mai utilizzato alcuno dei suindicati dispositivi. Tra coloro che hanno indicato di aver utilizzato tali presidi 16 hanno specificato l'ambito di utilizzo:

- 4 in occasione di gravi traumi della strada;

- 1 su ferite da armi da fuoco;

- 1 su ferite da taglio;

- 3 in occasione di amputazione di arto;

- 1 frattura di clavicola;

- 1 corpo estraneo;

- 1 ferita con motosega;

- 1 durante un servizio militare;

- $1 \mathrm{KED}$;

- 1 sospetto trauma arterioso;

- 1 non interpretabile;

Del campione in esame, 65 professionisti indicano di avere in dotazione ai mezzi di soccorso su cui lavorano un tourniquet di tipo militare, 5 un'emergency bendage, 17 delle medicazioni emostatiche, 67 un Crico Kit, 7 un needle kit, 6 una medicazione con valvola. La completa assenza di questi presidi è stata dichiarata da 51 intervistati. Indagando i fabbisogni formativi degli intervistati emerge che 21 persone ritengono di aver scarsa o nulla formazione in ambito di maxi-emergenza, 92 in tematiche CBRN, 21 relativamente all'approccio al trattamento sanitario obbligatorio (TSO) in supporto alle forze 
dell'ordine e 97 relativamente ad interventi in occasione di attacco terroristico, sparatoria o esplosione.

Domanda n. 8 Gestione trauma penetrante addome

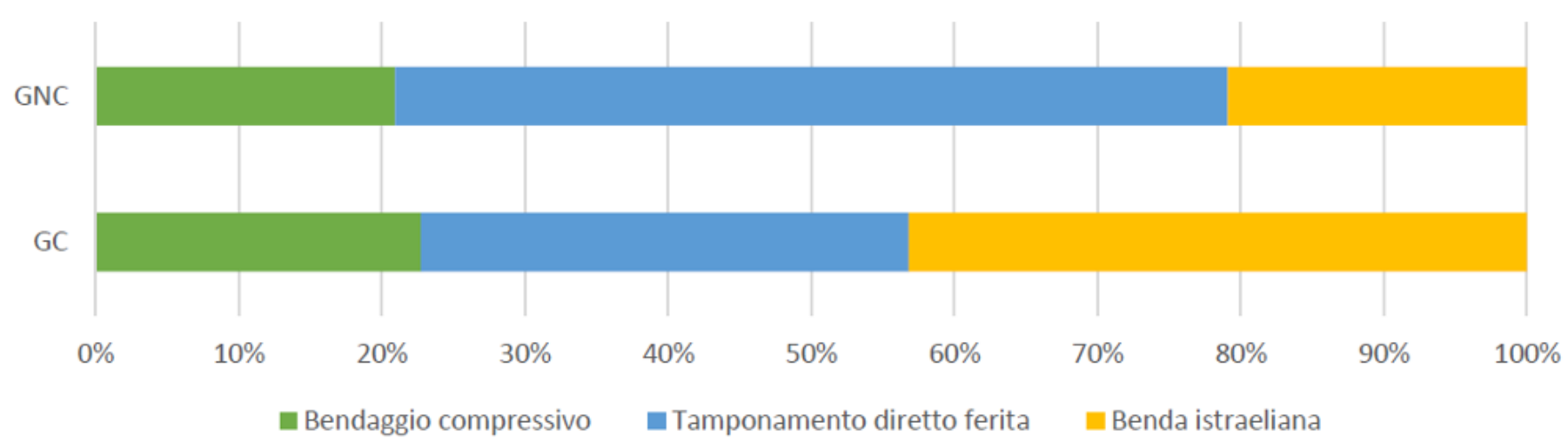

Domanda n.9 Gestione ferita penetrante torace

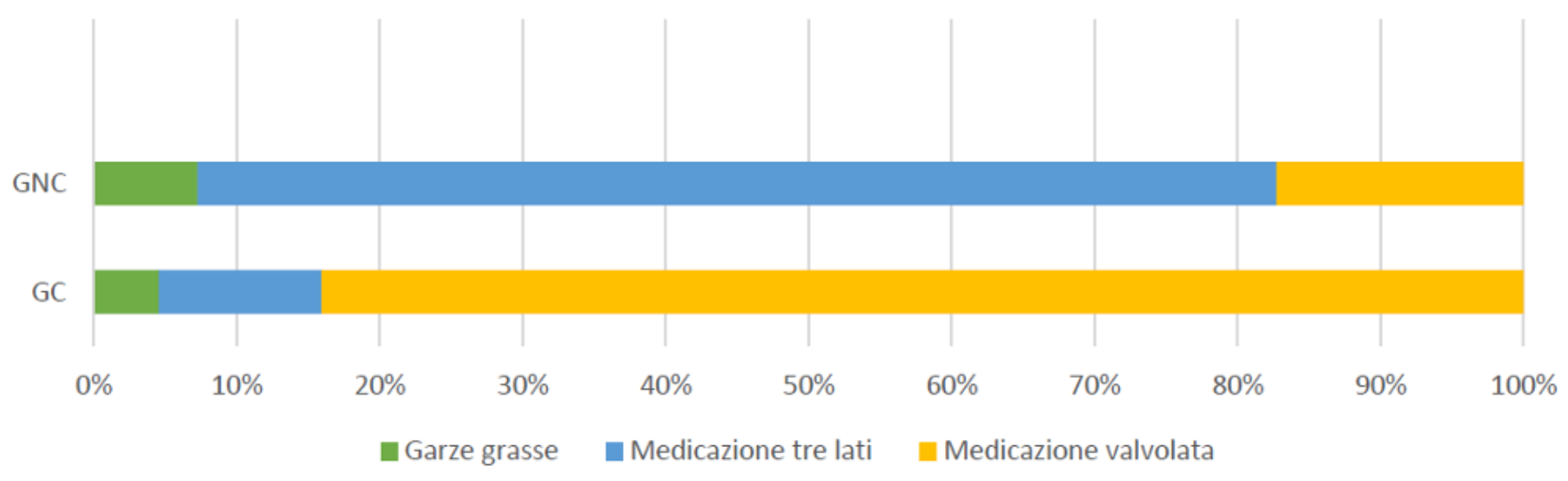

Domanda n. 10 Gestione pneumotorace chiuso

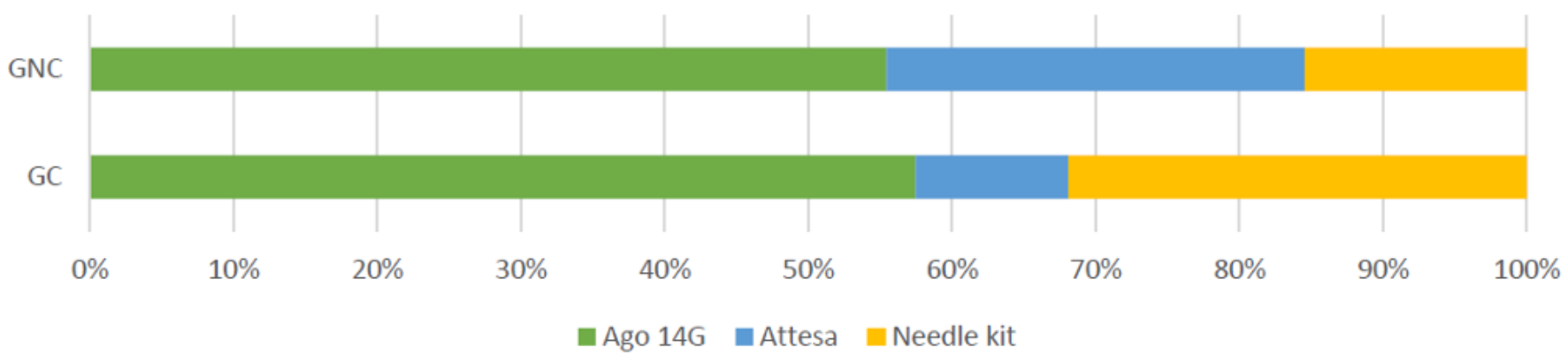




\section{Domanda n. 11 Gestione ostruzione vie aeree}

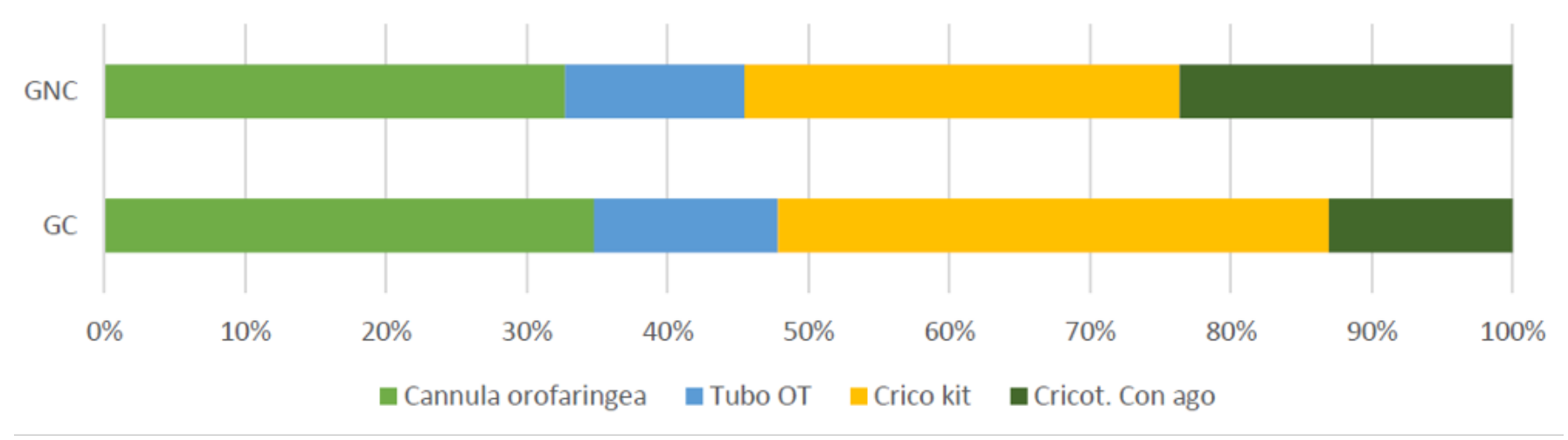

\section{Discussione e conclusione}

Allo studio hanno partecipato 154 professionisti operanti in servizi di emergenza ed urgenza medica. Il 53,2\% di questi appartengono alla categoria degli infermieri. L'anzianità lavorativa nel servizio emergenza urgenza è superiore a 10 anni per il 47,4\% del campione. Tale percentuale sommata al $21,4 \%$ di quelli che hanno risposto di avere una anzianità lavorativa compresa tra i 6 e i 10 anni compone un campione avente una prevalenza di professionisti con livelli di competenza distribuiti tra abili ed esperti. ${ }^{39}$ Solo il $27,9 \%$ del totale ha una formazione post-laurea avanzata di tipo universitario. Il $68.8 \%$ di chi ha compilato il questionario svolge la propria attività lavorativa nel Nord d'Italia. Il 71,4\% (110 partecipanti) degli intervistati non ha svolto corsi in ambito di medicina tattica mentre il restante $28,6 \%$, pari a 44 partecipanti, ha effettuato almeno un corso di formazione con questa tematica.

Confrontando le risposte del gruppo che ha effettuato i corsi di medicina tattica (GC) con quelle del gruppo che non ha effettuato tali corsi (GNC) relative alle core competences, ${ }^{40}$ emerge che nell'approccio al paziente traumatico solo il 22,7\% del GNC tende ad anteporre il controllo dell'emorragia massiva al controllo delle vie aeree contro il 56,8\% del GC. Nella gestione dell'emorragia massiva di un arto non si evidenziano sostanziali differenze di trattamento in quanto le percentuali sono similmente ripartite e l'uso del tourniquet viene adottato precocemente nel 52,3\% del GC e nel $40,9 \%$ del GNC. Nella gestione del trauma penetrante all'addome, il 58,1\% dei GNC applicherebbe un tamponamento diretto sulla ferita contro il 34\% del GC. Il 43,1\% degli appartenenti al GC posizionerebbe un'emergency bendage mentre il 22,9\% un bendaggio compressivo.

Per la gestione del trauma penetrante al torace, il 95,5\% del gruppo GC indica l'utilizzo di una medicazione con valvola (88\%) o chiusa su tre lati (12\%), mentre il $75,5 \%$ del GNC 
utilizzerebbe una medicazione chiusa sui tre lati contro un $17,2 \%$ che indica una medicazione con valvola. In presenza di pneumotorace chiuso il GNC utilizzerebbe un ago da $14 \mathrm{~g}$ nel 55,5 \% dei casi, contro il 61,3\% del GC; sempre nel gruppo GC, il 27,7\% ha scelto come opzione un needle kit specifico per pneumotorace. Da segnalare che il $29 \%$ del GNC opterebbe per un approccio di attesa nella gestione dello pneumotorace contro $11 \%$ del GC. Per quanto riguarda la gestione dell'ostruzione delle vie aeree, il 54,5\% del GNC agirebbe reperendo un accesso d'urgenza mediante cricotiroidotomia utilizzando un Crico Kit nel $30,9 \%$ o un ago cannula nel $23,6 \%$. Il GC effettuerebbe tale manovra ( $54,5 \%$ degli intervistati) ma nel 40,9\% con il crico kit e nel 13,6\% con ago. Suddividendo ulteriormente il campione dei partecipanti in gruppi omogenei per competenze di base (medici, infermieri, autisti soccorritori) ed effettuando le stesse analisi non emergono sostanziali differenze percentuali nelle risposte. Il 30,5\% dei partecipanti del campione generale asserisce di aver usato un tourniquet di derivazione militare durante lo svolgimento del suo servizio ordinario, il 12,3\% una emergency bendage, il 13\% delle medicazioni emostatiche ed l'11\% un Crico Kit.

Per quanto riguarda la presenza di tali presidi nei mezzi di soccorso utilizzati dai professionisti partecipanti allo studio nel $42,2 \%$ è disponibile un tourniquet di tipo militare, nel 43,5\% un crico kit e solo nell'11\% delle medicazioni emostatiche. Il 63\% dei partecipanti allo studio si dichiara consapevole di avere scarsa o nessuna formazione nella gestione di un eventuale scenario terroristico, sparatoria o esplosione, mentre il 59,7\% dichiara di avere una carenza formativa relativamente alle tematiche NBCR. Interessante evidenziare che il $96 \%$ dei partecipanti dichiara voler partecipare ad una formazione nel caso fossero istituite delle squadre tattiche di emergenza medica e il $91 \%$ ne farebbe parte come operatore aderendo in modo volontario.

Così come evidenziato dalla letteratura, chi ha partecipato ad un corso di medicina tattica tende ad avere un approccio al paziente traumatizzato che assegna priorità al riconoscimento e controllo dell'emorragia massiva mediante l'utilizzo precoce di tourniquet o altri presidi specifici di derivazione militare se disponibili. In questo studio non si sono riscontrate sostanziali differenze nella gestione delle vie aeree se non per quanto riguarda la tipologia dei presidi; entrambe i gruppi hanno teso ad avere un approccio generale più conservativo e di attesa. Emerge una crescente applicazione delle linee guida TECC e dei presidi a derivazione militare anche nei sistemi di emergenza sanitaria ordinari in linea con quanto caldamente consigliato in letteratura. Come spesso accade in questa tipologia di studi, il campione analizzato ha espresso la necessità di ricevere formazione per essere in grado di fronteggiare al meglio eventi speciali che possono presentarsi in occasione di atti terroristici, di scontri a fuoco, dinamitardi o NBCR. Considerando quanto emerge dalla 
moderna letteratura scientifica, dai risultati ottenuti nei paesi che adottano questi protocolli all'interno dei propri servizi di emergenza sanitaria e tenendo conto degli innumerevoli campi applicativi di queste tematiche è auspicabile l'adozione di questi modelli in tutti i servizi di emergenza. Considerata la peculiarità di certe tipologie di intervento e la frequenza di presentazione, si ritiene meritevole di riflessione la possibilità di istituire, sull'esempio di alcuni stati esteri, squadre specialistiche in grado di intervenire a supporto dei sistemi di emergenza in occasione di particolari condizioni di crisi.

\section{Bibliografia}

1. Smeltzer S., Bare B., Hinkle J., Cheever K. Brunner-Suddarth Infermieristica medico-chirurgica. Quarta edizione Casa Editrice Ambrosiana; 2010.

2. Global Terrorism Database University of Maryland. Disponibile a: http://www.start.umd.edu/gtd. Ultimo accesso: 18 settembre 2018.

3. Massalou D., Ichai C., Mariage D., Baqué P. Terrorist attack in Nice - The experience of general surgeons. Journal of visceral surgery. 2018 Apr 28.

4. Edwards DS., McMenemy L., Stapley SA., Patel HD., Clasper JC. 40 years of terrorist bombings - A meta-analysis of the casualty and injury profile. Injury. 2016;47,3,646-52.

5. Boddaert G., Mordant P., Le Pimpec-Barthes F., Martinod E., Aguir S., Leprince P., Raux M., Couëtil JP., Fiore A., Lescot T., Malgras B., Pons F., Castier Y. Surgical management of penetrating thoracic injuries during the Paris attacks on 13 November 2015. European journal cardiothoracic surgery. 2017 Jun 1;51,6,1195-1202.

6. Bellamy, R.F. The causes of Death in Conventional Land Warfare: Implication for Combat Casualty Research. Military medicine. 1984;149,55-62.

7. Cooke T., Chesters A., Grier G. A systematic literature review of the pre-hospital lessons identified following mass casualty deliberate bombing incidents. Emergency medicine journal. 2017 Dec;34(12).

8. Turner CD., Lockey DJ., Rehn M. Pre-hospital management of mass casualty civilian shootings: a systematic literature review. Critical care. 2016 Nov 8;20,1,362.

9. National Association of Emergency Medical Technicians, corso TCCC. Disponibile a: http://www.naemt.org/education/naemt-tccc. Ultimo accesso: 18 settembre 2018.

10. National Association of Emergency Medical Technicians, Italia corso TCCC. Disponibile a: http://www.naemt-italia.it/tccc-tactical-combat-casualty-care/. Ultimo accesso: 18 settembre 2018.

11. National Association of Emergency Medical Technicians, corso TECC. Disponibile a: http://www.naemt.org/education/tecc. Ultimo accesso: 18 settembre 2018.

12. National Association of Emergency Medical Technicians Italia, corso TECC. Disponibile a: http://www.naemt-italia.it/tecc-tactical-emergency-casualty-care/. Ultimo accesso: 18 settembre 2018.

13. Committee for Tactical Emergency Casualty Care. Disponibile a: http://c-tecc.org/about/overview. Ultimo accesso: 18 settembre 2018. 
14. Williams M., Sizemore DC. Biologic, Chemical, and Radiation Terrorism Review. StatPearls Publishing. $2018-$ Apr 4.

15. Frank K. Butler MD. Two Decades of Saving Lives on the Battlefield: Tactical Combat Casualty Care Turns 20. Military medicine. 2017;182, 3/4,1563.

16.Thompson J., Rehn M., Lossius H. M., Lockey D. Risks to emergency medical of the medical literature. Critical care $2014 ; 18,521$.

17. Ferreri T.G., Weir A.J. EMS, Improvised Explosive Devices And Terrorist Activity. StatPearls Publishing. 2018 Jan.

18. Pérez C.U., Alonso V.G., Peiro L.O., Crespo J.M.G., López S.H. Implementation of the Hartford Consensus and Tactical Combat Casualty Care recommendations in emergency services: a review of the literature. Emergencias 2017; $29,416-421$.

19. Jacobs L.M., Wade D.S., McSwain N.E., Butler F.K., Fabbri W.P., Eastman A.L., Rotondo M., Sinclair J., Burns K.J. The Hartford Consensus: THREAT, A Medical Disaster Preparedness Concept. Journal of the american college of surgeons. $2013 ; 217,5,947-953$.

20. Callaway D.W. Translating Tactical Combat Casualty Care Lessons Learned to the High-Threat Civilian Setting: Tactical Emergency Casualty Care and the Hartford Consensus. Wilderness \& environmental medicine. 2017; 28, 140-145.

21. Cornelius B., Campbell R., McGauly P. Tourniquets in Trauma Care: A Review of Application. Journal of trauma nurse.2017;24,3,203-208.

22. Chatfield-Ball C., Boyle P., Autier P., Herzig van Wees S., Sullivan R. Lessons learned from the casualties of war: battlefield medicine and its implication for global trauma care. Journal of the royal society of medicine. 2015;108,3,93-100.

23. Redman T.T., Ross E. M. A novel expeditionary perfused cadaver model for trauma training in the out of hospital setting. The Journal of Emergency Medicine.2018;55,3,383-389.

24. Beaucreux C., Benoi^t V., Miles E., Ausset S., Pasquier P. Application of tourniquet in civilian trauma: Systematic review of the literature. Anaesthesia Critical Care \& Pain Medicine 2018; https://doi.org/10.1016/j.accpm.2017.11.017.

25. Bennett B.L., Bleeding Control Using Hemostatic Dressings: Lessons Learned. Wilderness \& environmental medicine.2017; 28, 39-49.

26. Bennett L., Littlejohn L. Review of New Topical Hemostatic Dressings for Combat Casualty Care. Military medicine.2014;179, 5:497.

28. Boulton A.J., Lewis C.T., Naumann D.N., Midwinter M.J. Prehospital haemostatic dressings for trauma: a systematic review. Emergency medicine J.2018;35,449-457.

29. Littlejohn L., Bennett B.L., Drew B. Application of Current Hemorrhage Control Techniques for Backcountry Care: Part Two, Hemostatic Dressings and Other Adjuncts. Wilderness \& environmental medicine.2015;26, 246-254.

30. Mabry R.L., Kharod C.U., Bennett B.L. Awake Cricothyrotomy: A Novel Approach to the Surgical Airway in the Tactical Setting. Wilderness \& environmental medicine,2017;28, 61-68.

31. Macêdo M.B., Bezerra guiMarães R., Martins riBeiro S., MaraBuco de sousa K.M..Emergency cricothyrotomy: temporary measure or definitive airway? A systematic review. Revista do colegio brasileiro de cirurgioes. 2016; 43,6,493-499.

32. Lewis P., Wright C. Saving the critically injured trauma patient: a retrospective analysis of 1000 uses of intraosseous access. Emergency medicine journal.2015;32,463-467. 
33. Petitpas F., Guenezan J., Vendeuvre T., Scepi M., Oriot D., Mimoz O. Use of intra-osseous access in adults: a systematic review. Critical care.2016;20,102.

34. Butler Jr. F. K. Fluid Resuscitation in Tactical Combat Casualty Care: Yesterday and Today. Wilderness \& environmental medicine.2017;28,74-81.

35. Hooper T.J., Nadler R., Badloe J., Butler F.K., Glassberg E. Implementation and execution of military forward resuscitation programs. Shock.2014;41, 1,90-97.

36. Ravi P.R., Puri B. Fluid resuscitation in haemorrhagic shock in combat casualties. Disaster and military medicine.2017;3,2.

37. Fisher A.D., Miles E.A., Cap A.P., Strandenes G., Kane S.F. Tactical Damage Control Resuscitation. Military medicine.2015;180, 8,869.

38. Piattaforma di elaborazione questionari on line. Disponibile a: www.survio.com. Ultimo accesso: 18 settembre 2018.

39. Benner P. From novice to expert, The American Journal of Nursing. 1982;82(3):402-407.

40. Pennardt A. M., Callaway D.W., Kamin R., Llewellyn C.H., Shapiro G. L., Carmona R. H., Schwartz R. Integration of tactical emergency casualty care into the National Tactical Emergency medical support competency domains. Journal of Special Operations Medicine. 2016;16(2):62-66.

41. Rotondo MF, Schwab CW, McGonigal MD, et al., Damage control: an approach for improved survival in exsanguinating penetrating abdominal injury. J Trauma, 1993. 35(3): p. 375-82; discussion 382-3.

42. Holcomb, JB, Jenkins, D, Rhee, P, et al., Damage control resuscitation: directly addressing the early coagulopathy of trauma. J Trauma, 2007. 62(2): p. 307-10.

43. Bjerkvig CK, Strandenes G, Eliassen HS, et al., "Blood failure" time to view blood as an organ: How oxygen debt contributes to blood failure and its implications for remote damage control resuscitation. Transfusion, 2016.56 Suppl 2: p. S182-9.

44. Woolley T, Thompson P, Kirkman E, et al. Trauma Hemostasis and Oxygenation Research Network position paper on the role of hypotensive resuscitation as part of remote damage control resuscitation. J Trauma Acute Care Surg, 2018 Jun;84(6S Suppl 1):S3-S13.

45. American College of Surgeons, Advanced Trauma Life Support (ATLS), $10^{\text {th }}$ Edition 2018. https://viaaerearcp.files.wordpress.com/2018/02/atls-2018.pdf Accessed Apr 2019. 